\title{
ESTUDO DO EQUILÍBRIO E DA DINÂMICA DA BIOSSORÇÃO DO ÍON CÁDMIO PELA MACRÓFITA LEMNA MINOR EM COLUNA DE LEITO FIXO
}

\author{
Marielen Cozer Ribas \\ Rafael Canevesi ${ }^{2}$ \\ Edson Antonio da Silval
}

\begin{abstract}
Resumo: Este trabalho objetivou avaliar a eficiência da biomassa in natura e pré-tratada Lemna minor na remoção do cádmio de soluções sintéticas. O pré-tratamento da macrófita foi realizado utilizando soluções de hidróxido de sódio e ácido sulfúrico $0,1 \mathrm{M}$ e 0,01M, separadamente. Tendo o pré-tratamento com hidróxido de sódio $0,1 \mathrm{M}$ apresentado melhores resultados. Foram realizados experimentos para a obtenção dos dados de equilíbrio em sistema batelada e avaliação da dinâmica da remoção do cádmio em colunas de leito fixo. Os dados experimentais de equilíbrio foram obtidos na temperatura de $30^{\circ} \mathrm{C} \mathrm{e} \mathrm{pH}$ inicial de 5 . Os ensaios de remoção de cádmio na coluna de leito fixo foram realizados em 5 vazões volumétricas distintas, 5, 6, 7, 8 e $9 \mathrm{~mL} / \mathrm{min}$. Na modelagem dos dados de equilíbrio foram utilizados os modelos de Isotermas de Langmuir, Freundlich e Sips. A isoterma de Sips foi a que melhor representou os dados experimentais de equilíbrio. A capacidade máxima de remoção foi de $0,861 \mathrm{meq} / \mathrm{g}$ obtida em reator batelada. Para a modelagem da dinâmica de remoção do $\mathrm{Cd}$ na coluna de leito fixo foi utilizado um modelo que leva em consideração a transferência de massa na fase sólida. A taxa de adsorção foi descrita utilizando um modelo de força motriz linear (FML). O modelo foi capaz de descrever satisfatoriamente o comportamento das curvas de ruptura experimentais.
\end{abstract}

Palavras-chave: biossorção, cádmio, Lemna minor

Abstract: This study aimed to evaluate the Lemna minor in natura and pretreated biomass efficiency in the cadmium removal from synthetic solutions. The macrophyte pretreatment was performed using sodium hydroxide and sulfuric acid solutions separately at $0,1 \mathrm{M}$ and $0,01 \mathrm{M}$. The sodium hydroxide solution $(0,1 \mathrm{M})$ pretreatment achieved the best outcome. In addition, there were carried out experimental tests in order to obtain equilibrium data from a batch system and evaluation of the cadmium removal dynamics in a fixed bed column. Experimental equilibrium data were obtained at $30^{\circ} \mathrm{C}$ and initial $\mathrm{pH}$ at 5 . Cadmium removal tests were performed in a fixed bed column at five different flow rates: 5, 6, 7, 8 and $9 \mathrm{~mL} / \mathrm{min}$. Langmuir, Freundlich and Sips models were applied to equilibrium data modeling. Sips isotherm fitted best the experimental equilibrium data. Maximum removal capacity obtained by the batch reactor system was equivalent to $0,861 \mathrm{meq} / \mathrm{g}$. For the cadmium removal dynamics modeling there was applied a model that takes into account mass transfer on solid phase. The rate of adsorption was described using a linear driving force model (FML).The applied model described adequately the experimental rupture curves behavior.

Keywords: biosorption, cadmium, Lemna minor

\footnotetext{
1 Programa de Pós-graduação Stricto Sensu em Engenharia Química da Universidade Estadual do Oeste do Paraná - UNIOESTE. E-mail: marielencozer@yahoo.com.br.

2 Graduação em Engenharia Química pela Universidade Estadual do Oeste do Paraná - UNIOESTE.

E-mail: rafael_canevesi@hotmail.com.
} 


\section{INTRODUÇÃO}

As águas residuais industriais contaminadas com metais pesados contribuem para este problema ambiental, oferecendo perigo a saúde pública e ao ambiente quando despejado sem tratamento adequado no corpo receptor.

Os resíduos contaminados por metais pesados são mais difíceis de serem tratados do que outros tipos de resíduos, necessitando na maioria dos casos, para adequação ambiental do efluente, de tratamentos mais específicos. (MONTEIRO, 2010). A maioria dos sais de metais pesados é solúvel em água e consequentemente não pode ser separado por processos físicos convencionais.

O cádmio vem sendo descrito como um dos elementos mais perigosos de todos os metais contaminantes presentes nos alimentos e no ambiente do homem, não apenas pela sua toxicidade, mas também pela larga distribuição e aplicações industriais (ALBETINI et al., 2007).

Atualmente há grande interesse em encontrar métodos eficientes de tratamento de efluentes que contenha metais pesados antes do descarte no meio ambiente. Segundo Silva et al. (2009), os métodos convencionais mais utilizados para a remoção de metais pesados, são processos químicos como a oxidação química, precipitação química e redução química. Estes métodos nem sempre são eficientes e geralmente apresentam custo elevado além de gerar resíduos sólidos, necessitando assim de uma nova etapa de tratamento.

Uma das alternativas é o uso de biossorventes com o fim de remover ou isolar substâncias tóxicas que estejam presentes nos efluentes domésticos ou industriais. Alguns estudos realizados (SCHENEIDER,1995; SCHNEIDER E RU$\mathrm{BIO}, 1999)$ indicam que biomassas de macrófitas aquáticas, mesmo secas, possuem alta capacidade de acumular íons metálicos. A biomassa Lemna minor tem apresentado bons resultados.

A Lemna minor é uma planta de tamanho reduzido comparado com outras macrófitas flutuantes de água doce. É provida apenas de folhas solitárias, ou em grupos de 2 a 4, contendo uma única raiz não ramificada (LORENZI, 1991), e consequentemente sua quantidade de massa por área é menor que de outras espécies. Esta espécie se reproduz por sementes e, principalmente, por meios vegetativos, a partir de brotos laterais que se soltam da planta mãe (KLEIN e AMARAL, 1988).
VOLESKY (1990) sugere vários tipos de configuraçoóes de reatores para a remoção de metais usando partículas ou pó de biomassas ativas. Reatores de tanques agitados contínuos são úteis quando o biossorvente em pó é utilizado. Sistemas de leito fluidizado que operam de modo contínuo requerem altas velocidades de escoamento para manter as partículas do biossorvente em suspensão (MURALEEDHARAN et al., 1991)

Segundo Seolatto et al. (2009), as colunas de leito fixo são uma das configurações mais eficientes para processos contínuos de remoção de metais pesados, permitindo melhor aproveitamento do biossorvente devido a seus ciclos de regeneração, e também oferece como vantagens: o pequeno espaço, a simples operação, a possibilidade de tratamento de grandes volumes de efluentes de forma contínua.

O comportamento de troca em um leito fixo baseia-se nas zonas de transferência de massa (ZTM). Este conceito corresponde a uma "macro" aproximação, estruturada no movimento da zona de transferência de massa durante a operação de sorção, sendo a ZTM considerada como a porção do leito na qual o componente iônico presente na solução de alimentação é transferido para a fase sólida do sistema. Como resultado deste tratamento, obtém-se um método completo de projeto englobando equilíbrio e a cinética de transferência de massa. Este método é aplicável ao sistema quando há uma rápida formação de uma zona estável de transferência de massa, a qual progride através do leito durante a etapa de sorção/troca e quando a taxa de remoção de íons é muito rápida. Em decorrência destes fatos tem-se que o processo de troca/adsorção é restrito, preferivelmente, a uma regiáo limitada do leito, a qual, dentro de um curto período de tempo após o início do processo, flui progressivamente a uma taxa constante. Este deslocamento é determinado pela vazão de operação, concentração de soluto e a capacidade específica de troca do adsorvente (GEANKOPLIS, 1993).

São vários os fatores que interferem na zona de transferência de massa, tais como: temperatura, vazão, relação de equilíbrio e parâmetros de construção da coluna. O tempo de contato é muito importante no projeto de colunas de adsorção, portanto a vazão volumétrica é um dos principais parâmetros no projeto de uma coluna de adsorção de leito fixo (McKAY e BINNO, 1990). 
Segundo Aksu e Kutsal (1998), a taxa de remoção de metais pelo processo de biossorção depende da transferência de massa e da velocidade na qual o sistema atinge o equilíbrio.

Um dos elementos mais importantes associados ao projeto de uma coluna de adsorção de leito fixo é pré-determinar quando esta coluna alcançará o ponto de saturação para um dado conjunto de condições de um influente (WEBER e LIU, 1980).

A similaridade entre biossorção e troca iônica indica que a biomassa pode existir em diferentes formas iônicas e que o bioadsorvente envolvido na remoção e recuperação contínua de metais pode operar em diferentes ciclos iônicos. A forma iônica da biomassa pode afetar a capacidade de adsorção, a seletividade, a estabilidade química e as propriedades físicas do bioadsorvente. Além disso, um ciclo iônico influencia a eficiência e o grau de adsorção de uma coluna. Sendo assim, algumas vezes se faz necessário o pré-tratamento da biomassa antes da sua utilização (GASPAR, 2003).

Estudos realizados por SAYGIDER et al. (2005) na remoção individual dos metais Cd(II), $\mathrm{Cu}(\mathrm{II})$ e $\mathrm{Ni}$ (II) empregando a biomassa da macrófita Lemna minor. nas formas in natura e modificada por pré-tratamento químico com ácido sulfúrico e com hidróxido de sódio mostraram que esta espécie de macrófita tem elevada capacidade de biossorção de metais pesados e que os melhores resultados foram obtidos com a biomassa pré-tratada.

Neste trabalho, foi investigado a biossorção do cádmio pela macrófita Lemna minor em reator batelada para a obtenção dos dados de equilíbrio e em coluna de leito fixo. Os dados de equilíbrio foram descritos por diferentes modelos de isotermas de adsorção e a dinâmica da remoção do metal em coluna de leito fixo foi representada pelo modelo de força motriz linear (FML).

\section{MATERIAIS E MÉTODOS}

\subsection{PREPARAÇÃO DA BIOMASSA}

A macrófita aquática foi obtida em lagoas do Oeste do Paraná. Todo o material coletado foi lavado com água corrente, enxaguado com água deionizada e seco a $60^{\circ} \mathrm{C}$ até massa constante (COSSICH et al., 2004). Em seguida foi realizado o pré-tratamento de quatro parcelas da biomassa, cada uma com solução diferente. Foram utilizadas soluções de ácido sulfúrico e hidróxido de sódio, em concentrações de $0,01 \mathrm{M}$ e $0,1 \mathrm{M}$. O prétratamento da alga foi realizado colocando-se o material em contato com as respectivas soluções por $2 \mathrm{~h}$ sob agitação e temperatura controlada de $30^{\circ} \mathrm{C}$.

\subsection{EFEITO DO PRÉ-TRATAMENTO}

Para avaliar o efeito do pré-tratamento da biomassa na biossorção do cádmio foram realizados experimentos em batelada com duas concentrações de soluções de cádmio: $12 \mathrm{mg} / \mathrm{L}$ e 15 $\mathrm{mg} / \mathrm{L}$. Aproximadamente $0,3 \mathrm{~g}$ da biomassa nas formas in natura e pré-tratada foram colocadas em contato com cerca de $50 \mathrm{~mL}$ das diferentes soluções de cádmio numa mesa agitadora orbital com temperatura controlada de $30^{\circ} \mathrm{C}$. Após 48 horas, foi efetuada a separação da solução do biossorvente por meio de filtração a vácuo. Foram coletadas amostras e determinadas a concentração final e inicial de cádmio por espectrofotometria de absorção atômica. A quantidade removida de metal em cada amostra foi calculada a partir da seguinte equação:

$$
q_{j}^{*}=\frac{V\left(C_{j}^{0} \quad C_{j}^{*}\right)}{m_{s}}
$$

em que, $q_{j}^{*}$ é a concentração de equilíbrio do íon metálico $j$ retido pelo material biossorvente, $C_{j}^{0}$ é a concentração inicial do íon metálico $j$ na solução (meq/L), $C_{j}^{*}$ é a concentração de equilíbrio do íon metálico $j$ na solução (final do experimento) (meq/L), $V$ é o volume da solução (L) e $m_{s}$ a massa do biossorvente (base seca) (g).

\subsection{OBTENÇÃO DA ISOTERMA DE ADSORÇÃO EXPERIMENTAL EM REATOR BATELADA}

Os experimentos para a obtenção das isotermas foram realizados em reator batelada com temperatura e $\mathrm{pH}$ controlados $\left(30^{\circ} \mathrm{C}\right.$ e $\left.\mathrm{pH}=5\right)$. Soluçōes do íon cádmio com diferentes concentraçôes iniciais foram colocadas em contato com o bissorvente. Após 48 horas, as amostras foram filtradas.

As concentrações, inicial e de equilíbrio, das espécies metálicas presentes originalmente na 
solução, em cada frasco, foram determinadas por espectroscopia de absorção atômica e a concentração de equilíbrio, $q_{j}^{*}$, do íon metálico $j$ retido pelo material biossorvente, foi calculada pela Eq. (01).

\subsection{EXPERIMENTOS DE BIOSSORÇÃO EM COLUNAS DE LEITO FIXO}

Os experimentos foram realizados em uma coluna de leito fixo de aço inoxidável encamisa$\mathrm{da}$, com 2,7 cm de diâmetro interno. A coluna foi empacotada com $21 \mathrm{~g}$ de biomassa da macrófita Lemna minor (base seca) sendo, neste caso, altura do leito igual a $22,5 \mathrm{~cm}$. A solução de cádmio $\left(\mathrm{C}_{0}=0,63 \mathrm{meq} / \mathrm{L}, \mathrm{pH}=5\right)$, foi alimentada na coluna, com temperatura controlada de $30^{\circ} \mathrm{C}$ e previamente inundada com água deionizada. Cinco vazões foram testadas para avaliar o desempenho da operação: 5, 6, 7, 8 e $9 \mathrm{~mL} / \mathrm{min}$. Para cada ensaio, amostras foram coletadas no topo da coluna para se obter a curva de ruptura. As amostras foram, então, analisadas por espectrofotometria de absorção atômica.

\subsection{MODELO MATEMÁTICO DE EQUILÍBRIO DE ADSORÇÃO}

A isoterma de Langmuir é frequentemente empregada para descrever o comportamento do equilíbrio da adsorção em sistemas monocomponentes. É um modelo teórico que considera adsorção em monocamada e um número finito de sítios ativos (LANGMUIR, 1918; FAGUNDES-KLEN et al., 2007). A equação geral do modelo é dada por:

$$
q_{j}^{*}=\frac{q_{m} b_{j} C_{j}^{*}}{1+b_{j} C_{j}^{*}}
$$

em que: $b_{j}$ é a razão entre as constantes cinéticas de adsorção e dessorção (L/meq) e $q_{m}$ é a capacidade máxima do adsorvente (meq/g).

A isoterma de Freundlich é um modelo empírico que considera um adsorvente com superfície heterogênea e formação de multicamadas pelo soluto. $\mathrm{O}$ modelo de Freundlich possui a seguinte forma (FREUNDLICH, 1906):

$$
q_{j}^{*}=k C_{j}^{* n}
$$

em que: ké uma constante relacionada à capacidade de adsorção (meq/g), e $n$ é um parâmetro empírico relacionado à intensidade de adsorção.

A isoterma de Sips ou Langmuir-Freundlich, representada pela Equação 04, é um modelo empírico que leva em consideração a heterogeneidade da superfície do adsorvente e as interações entre as moléculas adsorvidas (TÓTH, 2001).

$$
q_{j}^{*}=\frac{q_{m} b_{j} C_{j}^{* n}}{1+b_{j} C_{j}^{* n}}
$$

em que: os parâmetros $q_{m}$ e $b_{j}$ possuem o mesmo significado que na isoterma de Langmuir. Já o parâmetro $n$ é um parâmetro empírico que indica a heterogeneidade do adsorvente. Quando $n=1$, o modelo se reduz à isoterma de Langmuir.

Os parâmetros das isotermas foram obtidos empregando o método Simplex Downhill (NELDER \& MEAD, 1965) minimizando a seguinte função objetivo.

$$
F=\frac{1}{n e} \sum_{i}^{n e}\left(q_{i}^{E X P}-q_{i}^{M O D}\right)^{2}
$$

Em que ne é o número de dados experimentais, $q_{i}^{E X P}$ e $q_{i}^{M O D}$ representam a concentração de equilíbrio do metal no biossorvente obtidas experimentalmente e calculada pelo modelo, respectivamente.

\subsection{CÁLCULO DA ZONA DE TRANSFERÊNCIA DE MASSA (ZTM)}

Para o cálculo da zona de transferência de massa, foram realizados experimentos para a curva de ruptura em 5 vazões distintas mantendo a concentração de alimentação fixa.

O comprimento da ZTM pode ser calculada pela equação 06 .

$$
Z T M=H_{t}-H_{u}
$$

Em que:

$$
\begin{aligned}
& H_{u}=\frac{q_{(t b)}}{q_{(t t)}} \cdot H_{t} \\
& q\left(t_{b}\right)=\frac{C_{0} \cdot \dot{Q}}{1000 \cdot m_{s}} \int_{0}^{t_{b}}\left(1-\frac{C}{C_{0}}\right) d t
\end{aligned}
$$


$q\left(t_{t}\right)=\frac{C_{0} \cdot \dot{Q}}{1000 \cdot m_{s}} \int_{0}^{t_{t}}\left(1-\frac{C}{C_{0}}\right) d t$

sendo $H_{t}$ a altura total do leito, $H_{u}$ a altura utilizada, $q_{(t b)}$ é a quantidade de metal removido até o tempo de ruptura $t_{b}$ que corresponde a tempo em que na saída da coluna $C_{\text {saida }} / C_{0}=0,05$ e $q_{(t)}$ é a quantidade de metal removida até a completa saturação do adsorvente, $\dot{Q}$ é a vazão volumétrica $\mathrm{em}^{3} /$ min e $m_{s}$ é a massa do adsorvente (base seca) em g.

\subsection{MODELO MATEMÁTICO}

Os modelos matemáticos são de suma importância no projeto de colunas de leito fixo, bem como de outros equipamentos. O modelo matemático no qual a taxa de adsorção é descrita utilizando um modelo de força motriz linear tem sido utilizado com sucesso para representar a dinâmica de adsorção/biossorção de metais em colunas de leito fixo utilizando diferentes adsorventes (OTERO et al., 2005; BORBA, 2006; SILVA et al., 2002; RUTHVEN, 1984).

O modelo matemático foi elaborado com base nas seguintes considerações: processo isotérmico e isobárico, porosidade do leito e propriedades físicas constantes, resistência a transferência de massa radial negligenciável, equilíbrio na interface sólido líquido. Como o modelo leva em conta a resistência à transferência de massa na fase sólida (modelo LDF), a força motriz para esta transferência é representada por uma função linear em relação à concentração do metal no adsorvente.

$\mathrm{O}$ balanço de massa para o metal na fase líquida é representado pela Equação 10:

$$
\frac{\partial C}{\partial t}+u_{0} \frac{\partial C}{\partial z}+\rho_{b e d} \frac{1}{\varepsilon} \frac{\partial q}{\partial t}-D_{L} \frac{\partial^{2} C}{\partial z^{2}}=0
$$

Onde $C$ é a concentração do metal no seio da fase fluida (meq/L), $q$ a concentração do metal na fase sólida (meq/g), $\varepsilon$ a porosidade do leito, $u_{0}$ a velocidade intersticial $(\mathrm{cm} / \mathrm{min}), \rho_{\text {bed }}$ a densidade do leito $(\mathrm{g} / \mathrm{L})$ e $D_{L}$ o coeficiente de dispersão axial $\left(\mathrm{cm}^{2} / \mathrm{min}\right)$.

A condição inicial é dada por:

$$
C(0, z)=0
$$

As condições de contorno usadas são de Dancwerts (1953).

$$
\begin{aligned}
& D_{L} \frac{\partial C}{\partial z}=u_{0}\left(C(t, 0)-C_{0}\right) \text { em } z=0 \\
& \frac{\partial C}{\partial z}=0 \text { em } z=L
\end{aligned}
$$

Para representar a taxa de adsorção foi considerado que a etapa de difusão no adsorvente controla o processo de transferência de massa. Neste caso, tem-se que $C_{e q}=C$ e a taxa de adsorção pode ser representada pela seguinte equação:

$$
\frac{\partial q}{\partial t}=-K_{S}\left(q-q_{e q}(C)\right)
$$

Onde o $\mathrm{q}_{\mathrm{eq}}$ será calculado a partir da isoterma de adsorção que apresentou maior eficiência em descrever os dados de equilíbrio em batelada.

A condição inicial para a equação da taxa é dada por:

$$
q(0, z)=0
$$

Neste trabalho apenas o parâmetro $K_{S}$ o coeficiente de transferência no sólido foi ajustado a partir dos dados experimentais da curva de ruptura.

O coeficiente de dispersão axial foi calculado pela equação proposta por OTERO et al. (2005), representada pela seguinte relação matemática:

$$
\frac{u_{0} d p}{D_{L}}=\left(0,2+0,11 \mathrm{Re}^{0,48}\right)
$$

Onde dp é o diâmetro médio da partícula, igual a $0,3356 \mathrm{~cm}$, Re é o número de Reynolds e $u_{0}$ é a velocidade intersticial.

Em que a variável $K_{S}$ obtidas a partir de otimização dos dados experimentais usando o método numérico Golden section search (EDGAR e HIMMELBLAU, 1989), usando como função objetivo o erro relativo, representado pela Equação 17. 
$F=\frac{1}{n e} \sum_{i}^{n e}\left(\frac{Y_{i}^{E X P}-Y_{i}^{M O D}}{Y_{i}^{E X P}}\right)$

Em que ne é o número de dados experimentais, $Y_{i}^{E X P}$ é o valor experimental da razão da concentração do metal na saída da coluna pela concentração de alimentação e $Y_{i}^{M O D}$ valor da razão de concentração calculada pelo modelo

\section{RESULTADOS E DISCUSSŌES}

\subsection{EFEITO DO PRÉ-TRATAMENTO}

A análise do efeito do pré-tratamento da biomassa foi realizada com base nos valores da concentração do metal removida pelas diferentes biomassas nas formas in natura e pré-tratada com ácido sulfúrico $0,01 \mathrm{M}$ e $0,1 \mathrm{M}$ e hidróxido de sódio nas mesmas concentrações, como mostra a Tabela 01.

Tabela 1. Valores da remoção do íon cádmio para os diferentes tratamentos da biomassa Lemna minor

\begin{tabular}{|c|c|c|c|}
\hline \multirow{2}{*}{$\begin{array}{c}\text { Pré- } \\
\text { Tratamento } \\
\text { da biomassa }\end{array}$} & \multirow{2}{*}{$\begin{array}{l}\text { Conc. } \\
\text { Solução } \\
\text { no pré- } \\
\text { tratamento }\end{array}$} & $\begin{array}{c}\text { Conc. } \\
\text { Inicial } \\
12 \mathrm{meq} / \mathrm{L}\end{array}$ & $\begin{array}{c}\text { Conc. } \\
\text { Inicial } \\
15 \mathrm{meq} / \mathrm{L}\end{array}$ \\
\hline & & $\begin{array}{c}\text { qeq } \\
(\mathrm{meq} / \mathrm{g})\end{array}$ & $\begin{array}{c}\text { qeq } \\
(\mathrm{meq} / \mathrm{g})\end{array}$ \\
\hline \multirow{2}{*}{$\begin{array}{l}\text { Biomassa } \\
\text { tratada } \\
\text { com Ácido } \\
\text { Sulfúrico }\end{array}$} & $0,01 \mathrm{M}$ & 0,20 & 1,30 \\
\hline & $0,1 \mathrm{M}$ & 0,18 & 0,81 \\
\hline \multirow{2}{*}{$\begin{array}{l}\text { Biomassa } \\
\text { tratada com } \\
\mathrm{NaOH}\end{array}$} & $0,01 \mathrm{M}$ & 0,25 & 1,36 \\
\hline & $0,1 \mathrm{M}$ & 0,58 & 1,47 \\
\hline $\begin{array}{c}\text { Biomassa in } \\
\text { natura }\end{array}$ & & 0,16 & 1,08 \\
\hline
\end{tabular}

Com os dados apresentados na Tabela 01, pode-se observar que o pré-tratamento com o hidróxido de sódio é mais eficiente em relação ao realizado com ácido sulfúrico. Porém, percebe-se também que indiferente ao pré-tratamento utilizado a remoção será mais eficiente do que nos ensaios com a biomassa in natura.

Constata-se ainda que o pré-tratamento com hidróxido de sódio $0,1 \mathrm{M}$ apresentou melhor resultado. Por este motivo, todos os resultados apresentados nas próximas seções correspondem a ensaios experimentais realizados com a biomassa pré-tratada nessa condição.

\subsection{ISOTERMAS DE ADSORÇÃO}

Neste trabalho, as isotermas de Langmuir, Freundlich e Sips foram utilizadas para analisar os dados experimentais obtidos.

$\mathrm{Na}$ Tabela 02 são apresentados os valores dos parâmetros obtidos com o ajuste das isotermas de adsorção aos dados experimentais de equilíbrio obtidos neste trabalho, bem como o erro médio relativo (AAD, Eq. 18) e o coeficiente de correlação para cada modelo.

$$
A A D=\frac{\left|q_{j}^{*}-q_{j}^{M O D}\right|}{q_{j}^{*}} \times 100
$$

em que: $q_{j}^{M O D}$ é o valor da quantidade adsorvida calculado pelo modelo.

Tabela 2. Valores dos parâmetros e análise estatística do ajuste das isotermas de adsorção

\begin{tabular}{|c|c|c|c|}
\hline Modelo & Parâmetros & $\begin{array}{l}\text { Erro } \\
\text { médio } \\
(\%)\end{array}$ & $\begin{array}{l}\text { Coef. de } \\
\text { correlação }\end{array}$ \\
\hline \multirow[t]{2}{*}{ Langmuir } & $\begin{array}{l}q_{m}=3,91 \\
(\mathrm{meq} / \mathrm{g})\end{array}$ & \multirow[t]{2}{*}{21,20} & \multirow[t]{2}{*}{0,72} \\
\hline & $b=0,056$ & & \\
\hline Freundlich & $\begin{array}{l}k=0,20 \\
N=0,96\end{array}$ & 23,46 & 0,62 \\
\hline \multirow{3}{*}{ Sips } & $\begin{array}{l}q_{m}=0,86 \\
(\mathrm{meq} / \mathrm{g})\end{array}$ & \multirow{3}{*}{5,67} & \multirow{3}{*}{0,97} \\
\hline & $\begin{array}{c}b=0,41 \\
(\mathrm{~L} / \mathrm{meq})\end{array}$ & & \\
\hline & $n=2,021$ & & \\
\hline
\end{tabular}

A partir da Tabela 02, pode-se verificar que o modelo de Sips foi o que melhor representou os dados de equilíbrio, pois apresentou maior coeficiente de correlação e menor erro médio. A capacidade máxima de remoção do cádmio estimada pelo modelo de Sips foi $0,86 \mathrm{meq} / \mathrm{g}$ e o valor do parâmetro $n$ indica que a superfície do biossorvente é altamente heterogênea.

Na Figura 01 são mostrados os dados experimentais, bem como as isotermas de adsorção ajustadas. 


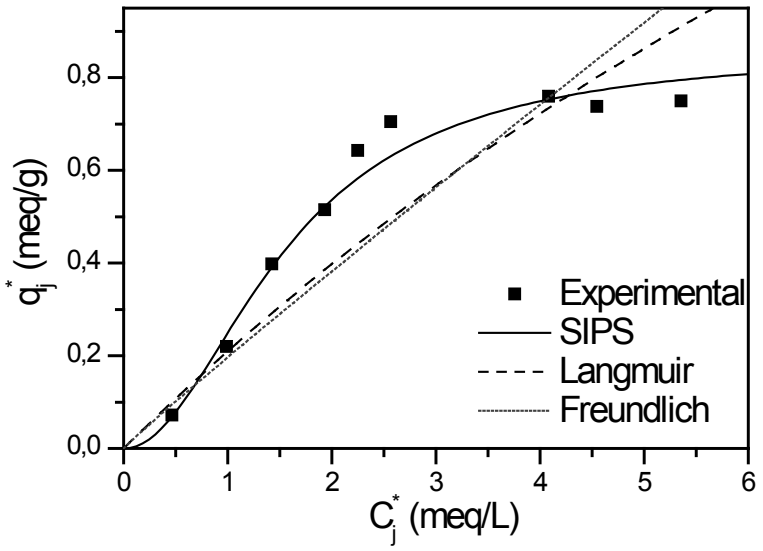

Figura 1. Isotermas de adsorção experimental e teórica da biossorção de cádmio por Lemna minor

Outros autores obtiveram valores similares de capacidade de biossorção com diferentes tipos de biossorvente e seus resultados são comparáveis aos encontrados no presente estudo, estes valores serão apresentados na Tabela 3.

Tabela 3. Comparativo entre vários materiais adsorventes na remoção do íon cádmio

\begin{tabular}{|c|c|c|}
\hline Adsorvente & Autor & $\begin{array}{c}\text { Remoção } \\
\text { Máxima Cd } \\
\text { (meg/g) }\end{array}$ \\
\hline Lemna minor & & 0,86 \\
\hline Eichhornia crassipes & Mishra (2008) & 0,62 \\
\hline Pistia stratoites & Mishra (2008) & 0,78 \\
\hline Spirodela polyrrhiza & Mishra (2008) & 0,50 \\
\hline Sargassum sp & $\begin{array}{c}\text { Sheng et al. } \\
(2004)\end{array}$ & 1,52 \\
\hline $\begin{array}{l}\text { Ulva sp sem } \\
\text { tratamento }\end{array}$ & $\begin{array}{l}\text { Suzuki et al. } \\
\text { (2005) }\end{array}$ & 1,10 \\
\hline $\begin{array}{l}\text { Ulva sp pré-tratada } \\
\text { com solução ácida }\end{array}$ & $\begin{array}{l}\text { Suzuki et al. } \\
\text { (2005) }\end{array}$ & 0,76 \\
\hline $\begin{array}{c}\text { Ulva sp pré-tratada } \\
\text { com solução } \\
\text { alcalina }\end{array}$ & $\begin{array}{l}\text { Suzuki et al. } \\
\qquad(2005)\end{array}$ & 1,61 \\
\hline $\begin{array}{c}\text { Biomassa } \\
\text { da bactéria } \\
\text { Streptomyces rimosus }\end{array}$ & $\begin{array}{l}\text { Selatina et al. } \\
\qquad(2004)\end{array}$ & 1,15 \\
\hline Lodo anaeróbico & $\begin{array}{l}\text { Hawari et al. } \\
\text { (2006) }\end{array}$ & 1,06 \\
\hline $\begin{array}{l}\text { Bagaço de coco } \\
\text { modificado com } \\
\text { tiouréia }\end{array}$ & $\begin{array}{l}\text { Neto et al. } \\
\text { (2009) }\end{array}$ & 0,64 \\
\hline $\begin{array}{l}\text { Sargasssum } \\
\text { filipendula }\end{array}$ & Klen (2006) & 1,26 \\
\hline
\end{tabular}

\begin{tabular}{|c|c|c|}
\hline Egeria densa & $\begin{array}{c}\text { Pietrobelli } \text { et } \\
\text { al. }(2008)\end{array}$ & 1,28 \\
\hline $\begin{array}{c}\text { Saccharomyces } \\
\text { cerevisiae }\end{array}$ & $\begin{array}{c}\text { Ferreira } \text { et al. } \\
(2007)\end{array}$ & 1,74 \\
\hline Amberlite IR-120 & Lee (2006) & 2,70 \\
\hline
\end{tabular}

Ao se comparar com outros adsorventes, percebe-se que mesmo não sendo o que apresenta maior remoção os valores obtidos são próximos demonstrando a eficiência da macrófita Lemna minor. Ressalta-se que a macrófita aqui estudada é de água doce, enquanto que algumas utilizadas para o comparativo são marinhas, o que pode representar maior dificuldade para obtenção e altos custos com transporte dependendo da região em que será utilizada.

\subsection{EXPERIMENTOS EM COLUNA DE LEITO FIXO - MODELAGEM DA DINÂMICA DE ADSORÇÃO}

Os modelos matemáticos são de suma importância no projeto de colunas de leito fixo, bem como de outros equipamentos.

A partir das curvas de ruptura experimentais pode-se calcular a capacidade de saturação da coluna, desde que os experimentos sejam conduzidos até a condição de saturação, isto é, quando a concentração das espécies químicas na saída seja igual a da alimentação.

De acordo com Ko (2001), Yoshida et al. (1994) e Wang e Weber (1993), a capacidade de remoção de íons metálicos em processo batelada e em processo contínuo são diferentes, além de que os resultados obtidos em batelada não podem descrever satisfatoriamente o comportamento do sistema contínuo.

O valor da capacidade de remoção do cádmio pelo biossorvente $\left(\mathrm{q}_{\mathrm{m}}\right)$ calculado a partir dos dados experimentais da coluna foi de $0,41 \mathrm{meq} / \mathrm{g}$ para a vazão de $9 \mathrm{~mL} / \mathrm{min}$, na qual ocorreu a saturação do biossorvente. $\mathrm{Na}$ concentração de 0,63 $\mathrm{meq} / \mathrm{L}$ (concentração de alimentação da coluna), o valor estimado para a remoção pela isoterma de adsorção de Sips foi de 0,111 meq/g. Verifica-se que a capacidade de remoção experimental do cádmio na coluna é maior que a prevista pela isoterma, isto se deve ao fato de que os dados experimentais de equilíbrio utilizados para o levantamento das isotermas foram obtidos em sistema fechado e batelada. Por se tratar de um processo 
de troca iônica, neste tipo de sistema, os íons liberados pelo biossorvente ficam presentes na solução, além disso, durante os ensaios de sorção foram adicionados íons na forma de soluções ácidas para ajustar o $\mathrm{pH}$ inicial no valor desejado (5).

Os dados de equilíbrio do cádmio foram tratados utilizando isotermas de adsorção. Nesta abordagem os efeitos dos íons liberados não são levados em conta. Portanto, esse efeito poderia ser uma justificativa para os desvios da capacidade de remoção do íon cádmio. A condição de equilíbrio na coluna é caracterizada pela ausência de íons liberados pelo biossorvente, pois o sistema é aberto.

Trabalhos encontrados na literatura, como os de Wang e Weber (1993), Yoshida et al., (1994), Silva et al. (2002) e Preetha e Viruthagiri (2007) demonstraram que os ensaios em batelada e em coluna resultam em diferentes capacidades de remoção de íons metálicos.

Para a obtenção da relação de equilíbrio, empregou-se o modelo de isoterma que melhor descreveu os dados experimentais de equilíbrio em batelada, e seus parâmetros apresentados anteriormente.

A partir dos dados experimentais da curva de ruptura obteve-se o parâmetro de transferência de massa na fase sólida $\left(K_{S}\right)$. Os resultados obtidos para esse parâmetro e o valor calculado para a dispersão axial, através da Eq.16, para as diferentes vazões são apresentados na Tabela 04 .

Tabela 4. Parâmetros obtidos pelo modelo matemático LDF e valores calculados para o coeficiente de dispersão axial

\begin{tabular}{|c|c|c|c|}
\hline $\begin{array}{c}\text { Vazão } \\
(\mathrm{mL} / \mathrm{min})\end{array}$ & $\begin{array}{c}K_{S}\left(\mathrm{~min}^{-1}\right) \\
10^{3}\end{array}$ & $\begin{array}{c}D_{L} \\
\left(\mathrm{~cm}^{2} / \mathrm{min}\right) \\
10^{4}\end{array}$ & $\begin{array}{c}\text { Função } \\
\text { Objetivo } \\
10^{2}\end{array}$ \\
\hline 5 & 1,5 & 1,5 & 0,55 \\
\hline 6 & 2,3 & 1,79 & 2,40 \\
\hline 7 & 4,55 & 2,09 & 8,53 \\
\hline 8 & 3,05 & 2,68 & 8,70 \\
\hline 9 & 4,61 & 2,66 & 5,26 \\
\hline
\end{tabular}

Através dos dados da Tabela 04 verifica-se que houve variação do coeficiente de transferência de massa no sólido $\left(K_{S}\right)$. O resultado da modelagem pode ser visualizado nas Figuras 02, 03, 04, 05 e 06.

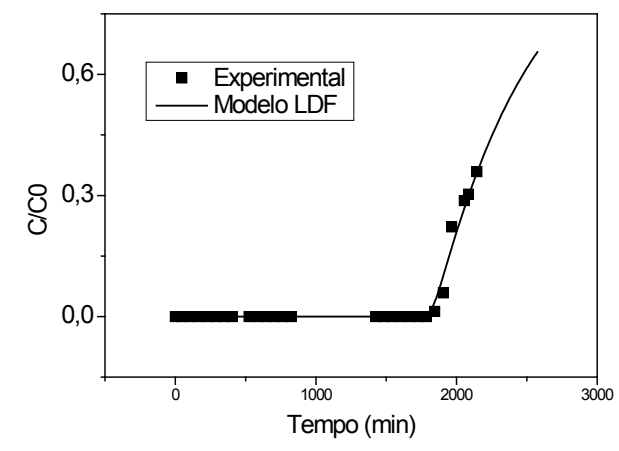

Figura 2. Curva de ruptura experimental obtida pelo modelo LDF para a vazão $5 \mathrm{~mL} / \mathrm{min}$

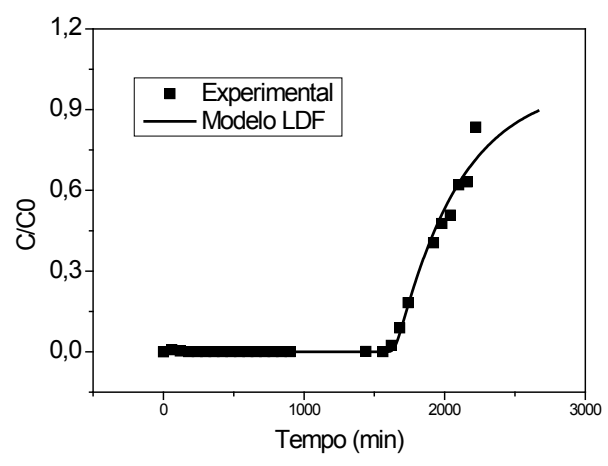

Figura 3. Curva de ruptura experimental obtida pelo modelo LDF para a vazão $6 \mathrm{~mL} / \mathrm{min}$

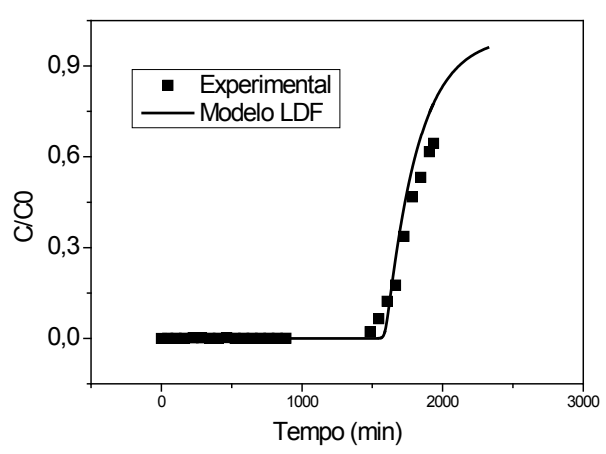

Figura 4. Curva de ruptura experimental obtida pelo modelo LDF para a vazão $7 \mathrm{~mL} / \mathrm{min}$

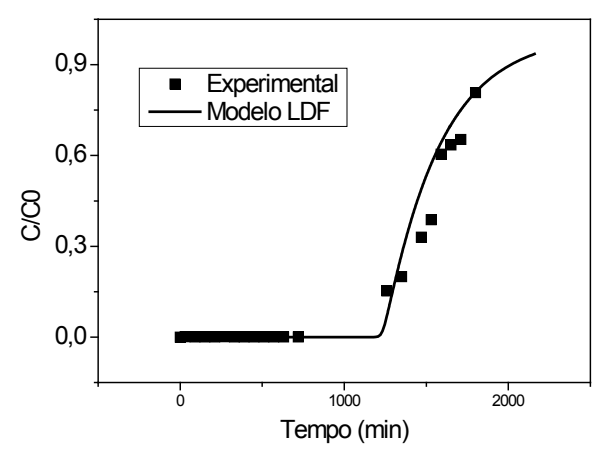

Figura 5. Curva de ruptura experimental obtida pelo modelo LDF para a vazão $8 \mathrm{~mL} / \mathrm{min}$ 


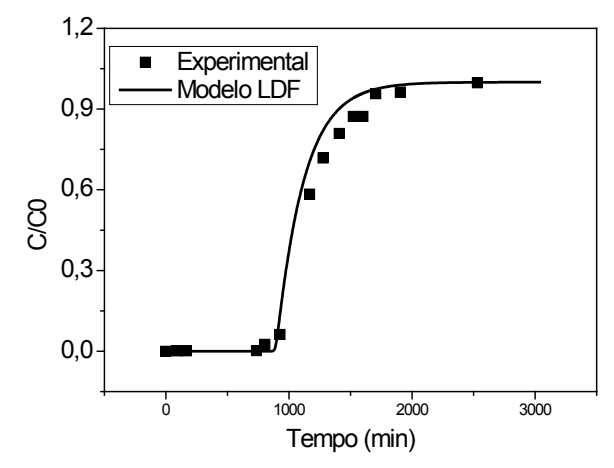

Figura 6. Curva de ruptura experimental obtida pelo modelo LDF para a vazão $9 \mathrm{~mL} / \mathrm{min}$

Os baixos valores da função objetivo e os gráficos apresentados mostram que o modelo conseguiu descrever a curva de ruptura de forma eficiente.

\subsection{EXPERIMENTOS EM COLUNA DE LEITO FIXO - CÁLCULO DA ZONA DE TRANSFERÊNCIA DE MASSA (ZTM)}

Foi calculado o tempo de ruptura considerando o seguinte valor da concentração adimensional $C / C_{0}=0,05$. A partir dos dados experimentais da curva de ruptura foi possível estimar as quantidades de metal removido até o ponto de ruptura $\mathrm{q}\left(\mathrm{t}_{\mathrm{b}}\right)$ e a quantidade removida até a saturação $\mathrm{q}\left(\mathrm{t}_{\mathrm{t}}\right.$. A Zona de Transferência de Massa foi calculada através da Equação 06. Como a concentração foi mantida constante em todos os experimentos na coluna de leito fixo, pode admitir que a quantidade de metal removida até a saturação é igual para todas as vazóes. Sendo assim, o valor $\mathrm{q}\left(\mathrm{t}_{\mathrm{t}}\right)$ é de $0,414 \mathrm{meq} / \mathrm{g}$ calculado para a vazão de 9 $\mathrm{mL} / \mathrm{min}$, onde ocorreu a saturação.

Os resultados para o tempo de ruptura e a ZTM são apresentados na Tabela 06.

A vazão é o principal parâmetro no projeto de uma coluna de leito fixo, pois, nos casos em que o processo de adsorção é controlado pela resistência à transferência de massa no interior da partícula, baixas vazôes volumétricas resultam em elevados tempos de residência permitindo que ocorra a adsorção, consequentemente aumentando a quantidade adsorvida na fase estacionária.
Tabela 5. Valores calculados para o tempo de ruptura e zona de transferência de massa nas diferentes vazões

\begin{tabular}{|c|c|c|}
\hline $\begin{array}{c}\text { Vazão } \\
(\mathrm{mL} / \mathrm{min})\end{array}$ & $\begin{array}{c}\text { Tempo de } \\
\text { Ruptura } \\
(\mathrm{min})\end{array}$ & $\begin{array}{c}\text { Zona de Transferência } \\
\text { de Massa } \\
(\text { ZTM em cm })\end{array}$ \\
\hline 5 & 1893,6 & 5,58 \\
\hline 6 & 1644 & 4,98 \\
\hline 7 & 1523,7 & 3,83 \\
\hline 8 & 896,3 & 9,08 \\
\hline 9 & 885,9 & 6,00 \\
\hline
\end{tabular}

Nos casos em que a transferência de massa externa é a etapa controladora, altas vazões volumétricas favorecem o decréscimo da resistência do filme líquido externo e o aumento do coeficiente de transferência de massa. Estes dois efeitos são antagônicos, a resposta do parâmetro vazão deve ser intermediária a estes dois extremos e devem ocorrer ao mesmo tempo (KO et al., 2000).

A vazão ótima de operação numa coluna de leito fixo é aquela em que obtêm-se o menor valor da zona de transferência de massa. Nas condições investigadas a vazão ótima de operação foi de 7 $\mathrm{mL} / \mathrm{min}$.

\section{CONCLUSÃO}

Observou-se que a biomassa da macrófita Lemna minor pré-tratada com solução de hidróxido de sódio $0,1 \mathrm{M}$ mostrou-se eficiente na remoção do cádmio. Na modelagem dos dados de equilíbrio da biossorção pelo emprego de Isotermas, o modelo de Sips foi o que melhor descreveu o comportamento dos dados experimentais. A capacidade máxima de remoção do cádmio foi de $0,861 \mathrm{meq} / \mathrm{g}$, determinada pelo modelo de Sips. O modelo matemático conseguiu descrever satisfatoriamente a dinâmica de remoção do íon cádmio em coluna de leito fixo. A vazão ótima de operação é de $7 \mathrm{~mL} / \mathrm{min}$ de acordo com a ZTM. Os resultados obtidos mostraram que a Lemna minor foi eficiente na remoção do cádmio e pode ser empregada no tratamento de efluentes em escala industrial que contenha este metal. 


\section{REFERÊNCIAS BIBLIOGRÁFICAS}

AKSU, Z. KUTSAL, T., (1998) Determinations of kinetic parameters in the biosorption of copper(II) on Cladophora sp., in a packed bed column reactor., Process Biochemistry, v.33, n.1, p-7-13.

ALBERTINI, S., CARMO, L.; PRADO FILHO, L. G. (2007). Determinação de isotermas de adsorção de Saccharomyces cerevisiae empregando acetato e sulfato de cádmio. Ciênc. Tecnol. Aliment., v. 27, n. 2, Campinas.

BORBA, C. E., (2006). Modelagem da remoção e metais pesados em coluna de adsorção de leito fixo. Faculdade de Engenharia Química, Universidade Estadual de Campinas, 145 p. Dissertação (Mestrado).

COSSICH, E. S., SILVA, E. A., TAVARES, C. R. G., CARDOZO FILHO, L., RAVAGNANI, T. M. K, (2004). Biosorption of Chromium(III) by Biomass of Seaweed Sargassum sp. in a Fixed-Bed Column, Adsorption, v. 10, p.129-138.

EDGAR, T. F; HIMMEBLAU, D. M. (1989). Optimization of chemical process. New York: McGraw-Hill.

FAGUNDES-KLEN, M. R.; FERRI, P.; MARTINS, T. D.; TAVARES, C. R. G.; SILVA, E. A. (2007). Equilibrium study of the binary mixture of cadmium-zinc ions biosorption by the Sargassum filipendula species using adsorption isotherms models and neural network. In. Biochemical Engineering Journal. v. 34, n.2, p. 136-146.

FERREIRA, J., CANUTO, M., SILVA, F., ALSINA, O., OLIVEIRA, L., CAVALCANTE, E., GOMES, W., \& MEDEIROS, M. (2007). Remoção do Íon Metálico Cádmio (Cd2+) Utilizando Resíduo da Indústria Alcooleira. Revista Eletrônica de Materiais e Processos.

FREUNDLICH, H. M. F. (1906). Über die adsorption in lösungen. In. Zeitschrift für Physikalische Chemie. v. 57, p. 385-470.

GASPAR, A. T. F. S., (2003). Bioadsorção de cromo em algas marinhas utilizando coluna extratora. Campinas: Faculdade de Engenharia Química,
Universidade Estadual de Campinas, 125 p. Dissertação (Mestrado).

GEANKOPLIS, C.J. (1993). Transport processes and unit operations. $4^{\mathrm{a}}$ ed., New York, PTR Prentice Hall, 1026 p.

HAWARI, A.H., MULLIGAN, C.N. (2006). Biosorption of lead (II), cadmium (II), copper (II) and nickel (II) by anaerobic granular biomass. Bioresource Technology, v. 97, pp. 692-700.

KLEN, M. R. F., (2006). Estudo do equilíbrio da biossorção monocomponente e multicomponente dos íons cobre, zinco e cádmio pela biomassa pré-tratada de alga marinha Sargassum filipendula, Universidade Estadual de Maringá, Maringá, Tese (Doutorado).

KLEIN, V.L.G., AMARAL, F. C. S. (1988). Plantas daninhas aquáticas flutuantes. Informe agropecuário, Belo Horizonte, v. 13, n. 150, p. 35-43.

KO, D. C. K., PORTER, J. F., McKAY, G. (2000). Optimesed correlations for the fixed bed adsorption of metal ions on bone char, Chem. Eng. Sci., v.55, p. 5819-5829.

KO, D. C. K., PORTER, J. F., McKAY, G. (2001). Film-pore diffusion model for the fixed bed sorption of copper and cadmium ions onto bone char, Wat. Res., v.35, p. 3876-3886.

LANGMUIR, I. (1918). The adsorption of gases on plane surfaces of glass, mica and platinum. Journal of the American Chemical Society. v. 40, n. 9, p. 1361-1403.

LEE, I. H., KUAN, Y. C., CHERN, J. M., (2006). Factorial experimental design for recovering heavy metals from sludge with ion-exchange resin. Journal of Hazardous Materials, v.138, p.549-559.

LORENZI, H. (1991). Plantas daninhas do Brasil: terrestres, aquáticas, parasitas, tóxicas e medicinais. Nova Odessa: Editora Plantarum, 2 ed.

MCKAY G., BINO M. J., (1990). Fixed bed adsorption for the removal of pollutants from water, Environmental Pollution, v. 66, p. 33-53. 
MISHRA, V. K., TRIPATHI, B. D., (2008). Concurrent removal and accumulation of heavy metals by the three aquatic macrophytes. Bioresource Technology, v.99, p. 7091-7097.

MONTEIRO, L., MAINIER, F.. (2010). Avaliação da utilização de esponjas e fios de aço na remoção de cobre de efluentes industriais. ENGEVISTA, Disponível em: http://www.uff.br/engevista/ seer/index.php/engevista/article/view/242/144. Acesso em: 20 Jan. 2011.

MURALEEDHARAN, T. R., IYENGAR, L. VENKOBACHAR, C. (1991). Biosorption: na atractive alternative for metal removal and recovery. Current Science, n.61, v.6, p.379-385.

NELDER, J. A.; MEAD, R. (1965). A Simplex Method for Function Minimization. The Computer Journal, v.7, p.308.

NETO, V. O. S., LIMA, A. C. A. , OLIVEIRAS A. G., NASCIMENTO, R. F., SOUZA, T. J. N. (2009). Remoção de cádmio por bagaço de coco modificado com tiouréa, 32a Reunião Anual da Sociedade Brasileira de Química.

OTERO, M., ZABKOVA, M., RODRIGUES, A. E., (2005). Adsorptive purification of phenol wastewaters: Experimental basis and operation of a parametric pumping unit. Chemical Engineering Journal v. 110, p.101-111.

PIETROBELLI, J. M. T. A., MÓDENES, A. N., SUZAKI, P. Y. R., ALFLEN, V. L., COLOMBO, A. (2008). Utilização da macrófita Egeria densa na biossorção do íon Cd(II). Simpósio de Inovação Tecnológica NIT - UNIOESTE.

PREETHA, B., VIRUTHAGIRI, T. (2007). Batch and continuous biosorption of chromium (VI) by Rhizopus arrhizus, Separation and Purification Technology, v. 57, p. 126-133.

RUTHVEN, D. M. (1994). Principles of adsorption and adsorption process, John Wiley \& Sons, New York, p.432.

SAYGIDER, S., GULNAZ, O., ISTIFLI, E. S., YUCEL, N., (2005). Adsorption of Cd(II), $\mathrm{Cu}(\mathrm{II})$ and $\mathrm{Ni}(\mathrm{II})$ ions by Lemna minor L.:Effect of physicochemical environment, Journal of Hazardous Materials B, v.126, pp.96-104.

SCHNEIDER, I.A.H. (1995). Biossorção de Metais Pesados com Biomassa de Macrófitos Aquáticos. Porto Alegre: Escola de Engenharia, 141p. Tese (Doutorado) - Programa de Pós-Graduação em Engenharia Metalúrgica e dos Materiais, UFRGS.

SCHNEIDER, I.A.H., RUBIO, J. (1999). Sorption of heavy metal ions by the non-living biomass of freshwater macrophytes. Environmental Science \& Technology, v.33, 2213-2217.

SELATINA, A., BAKHTI, M.Z., MADANI, A. KERTOUS, L., MANSOURI, Y., (2004). Biosorption of $\mathrm{Cd}^{+2}$ from aqueous solution by a $\mathrm{NaOH}$-treated bacterial dead Streptomyces rimosus biomass, Hydrometallurgy, v. 75, pp. 11-24.

SEOLATTO, A. A.; CÂMARA, M. M.; TAVARES, C. R., COSSICH, E. S.; SILVA, E. A. (2009). Remoção de níquel (II) de soluções aquosas pela biomassa Sargassum filipendula em múltiplos ciclos de sorção-dessorção. Acta Scientiarum Technology, v.31, n.1, p.57-64.

SHENG, P.X., TING, Y., CHEN, I.P., HONG, L., (2004). Sorption of lead, copper, cadmium, zinc and nickel by marine algal biomass: characterization of biosorption capacity and investigation of mechanisms. Journal of Colloid and Interface Science, v.275, pp. 131-141.

SILVA, E. A., COSSICH, E. S., TAVARES, C. R. G., CARDOZO, L., GUIRARDELLO, R. (2002). Modeling of copper (II) biosorption by marine alga Sargassum sp. in fixed bed, Process Biochem., v. 38, p. 791-799.

SILVA, A. F. M.; LIMA, I. O.; SILVA, J. A. G.; REIS, L. G., (2009). Estação Didática de Tratamento de Efluentes. Revista Eletrônica de Educação e Tecnologia do SENAI-SP. Vol. 3, n. 5.

SUZUKI, Y., KAMETANI, T. MARUYAMA, T., (2005). Removal of heavy metals from aqueous solution by nonliving Ulva seaweed as biosorbent, Water Research, v.39, pp.1803-1808. 
TÓTH, J. (2001). Adsorption, Theory, Modeling, and Analysis, Marcel Dekker Inc., $1^{\text {st }}$ edition.

VOLESKY, B. (1990). Biosorption of Heavy Metals. Boston. CRC Press, Inc., p 396.

WANG, C. K., WEBER Jr., W. J., (1993). Adsorption-desorption modeling of pollutants on granular activated carbon, J. Ksce v.13, p.279.
WEBER Jr., W. J., LIU, K. T. (1980). Determination of mass transport parameters for fixed bed adsorbers, Chem. Eng. Commun., v. 6, p. 49-60.

YOSHIDA, H., NISHIHARA, H. KATAOKA, T. (1994). Adsorption of BSA on strongly basic chitosan - Equilibria. Biotechnology and bioengineering, v. 43, p.1087-1093. 